\title{
Trauma Surgery $\&$ Acute Care Open \\ Evaluating trauma care, outcomes and costs in a system in crisis: the necessity of a Greek National Trauma Database
}

\author{
Apostolos Prionas (1) , 1,2,3 George Tsoulfas, ${ }^{3}$ Andreas Tooulias, ${ }^{3}$ Apostolos Papakoulas, ${ }^{3}$ \\ Athanasios Piachas, ${ }^{3}$ Vasileios Papadopoulos ${ }^{3}$
}

\begin{abstract}
- Additional material is published online only. To view please visit the journal online (http://dx.doi.org/10.1136/ tsaco-2019-000401).
\end{abstract}

'General Surgery, Queen's Hospital, Barking Havering and Redbridge Univesity Hospitals NHS Trust, Romford, London, UK ${ }^{2}$ Surgery and Cancer, Imperial College London, London, UK ${ }^{3}$ First General Surgery Department of Aristotle University of Thessaloniki, Papageorgiou General Hospital, Thessaloniki, Greece

Correspondence to Dr Apostolos Prionas; apostolosprionas@gmail.com

Received 21 October 2019 Revised 21 January 2020 Accepted 13 February 2020 (c) Author(s) (or their employer(s)) 2020. Re-use permitted under CC BY-NC. No commercial re-use. See rights and permissions. Published by BMJ.

To cite: Prionas $A$

Tsoulfas G, Tooulias A, et al.

Trauma Surg Acute Care Open 2020:5:e000401.

\section{ABSTRACT}

Background At present there is no organized trauma system in Greece and no national trauma database. The objective of this study was to record and evaluate trauma management at our university hospital and to measure the associated healthcare costs, while laying the foundations for a national database and the organization of regional trauma networks.

Methods Retrospective study of trauma patients ( $n=2320$ ) between 2014 and 2015, through our singlecenter registry. Demographic information, injury patterns, hospital transfer, investigations, interventions, duration of hospitalization, Injury Severity Score (ISS), outcomes, complications and cost were recorded.

Results Road traffic collisions (RTC) accounted for $23.2 \%$ of traumas. The proportion of patients who were transferred to the hospital by the National Emergency Medical Services decreased throughout the study $\left(n_{2015}=76 / 1192(6.38 \%), n_{2014}=109 / 1128(9.7 \%)\right)$ $(p<0.05) .1209(52.1 \%)$ of our trauma patients did not meet the US trauma field triage algorithm criteria. Overtriage of trauma patients to our facility ranged from $90.7 \%$ to $96.7 \%$, depending on the criteria used (clinical vs. ISS criteria). Ninety-one (3.9\%) of our patients received operative management. Intensive care unit admissions were 21 (0.1\%). Seventy-six (3.3\%) of our patients had ISS $>15$ and their mortality was $31.6 \%$. The overall non-salary cost for trauma management was $€ 623140.53 \%$ of these costs were attributed to RTCs. The cost resulting from the observed overtriage ranged from $€ 121000$ to $€ 315000$. Patients who did not meet the US trauma triage algorithm criteria accounted for $10.5 \%$ of total expenses.

Discussion Our results suggest that RTCs pose a significant financial burden. The prehospital triage of trauma patients is ineffective. A reduction of costs could have been achieved if prehospital triage was more effective.

Level of evidence Level IV.

\section{INTRODUCTION}

Trauma constitutes the 10th leading cause of death worldwide according to the WHO. ${ }^{1}$ In Greece, trauma is the fourth leading cause of death. Road traffic collisions (RTC) are over the EurA subregional (EurA is one of the three subregions that the European WHO region is divided into), the European Union (EU) and the USA average; the associated standardized death rate per 100000 of population, all ages included, is 14.6 (the equivalents are EurA: 13.3, EU: 9.3, USA: 12.4). ${ }^{12}$

Trauma databases have been established globally to improve trauma management by auditing trauma care. In the literature, most database publications come from the USA. Among European countries, Germany and the UK have the majority of publications. ${ }^{3}$ To date, there is no national trauma database in Greece. The annual epidemiology of trauma in our country can only be followed via police and emergency medical service (EMS) reports. ${ }^{45}$

There is no organized trauma system in Greece. Trauma management is provided by the Greek National Health System that has three levels of healthcare facilities. Primary healthcare units are general practitioner-based outpatient facilities, mostly in remote areas to address the need for emergency care and in cities to provide routine primary care. Minor trauma can be managed at this level of healthcare. Secondary healthcare units are the district general hospitals, which are situated in big cities. The third level of healthcare consists of specialized centers and university hospitals which are primarily located in major cities. Secondary healthcare units may function like a level II, III or IV trauma center, whereas a tertiary unit has capabilities similar to a level I or II trauma center. ${ }^{45}$

In financial terms, healthcare expenditure per capita in Greece is estimated at about $€ 1809$. The European average is €2193 and in the USA it is approximately US\$9146. ${ }^{67}$ During the national financial crisis the share of the gross domestic product (GDP) allocated to healthcare significantly decreased, from $9.76 \%$ of the GDP in 2009 to $8.75 \%$ of the GDP in 2013. At the same time, the GDP of Greece decreased by $20.2 \%$. As a result, the annual average growth rate in per-capita health expenditure (2009-2013) in the country decreased by $9 \%$.

The objective of this study is to record and evaluate trauma management at a university hospital in a major Greek city as well as to measure the associated healthcare expenses, while setting up one of the first trauma databases in the country. We also sought to suggest specific modifications to the National Health System to address the needs of trauma patients more effectively.

\section{METHODS}

The study was conducted between March 1, 2014 and October 31, 2015, at our university hospital. 
All data were gathered in an anonymized manner and there was no risk of personal data identification.

\section{Outline of the current operating system}

There are four tertiary university hospitals (level I/II trauma centers) and two district general hospitals (level III/IV trauma centers) that cover the trauma service for the city of Thessaloniki and the surrounding geographic regions (approximately 15\% of the country's population). Any given day, only one tertiary university hospital and one district general hospital receive trauma and emergency cases. This results in every tertiary hospital accepting new trauma/emergency cases once every 4 days and every district general hospital once every 2 days. In every tertiary university hospital, there are multiple departments covering the same specialty, that is, in our hospital there are three general surgery departments, two orthopedic departments, and so on. The departments covering the same specialty receive new cases in rotation, when the index hospital is accepting trauma and emergencies. Patients can be brought to hospital by the Greek National Medical Services (GNMS) (the national prehospital provider). Alternatively, they can self-present and register to the emergency department without need for any previous triage or referral.

\section{Physical and human resources}

Our university hospital has 800 beds. There are 14 operating rooms, a postoperative care area (recovery), one intensive care unit (ICU) with 16 beds and one emergency department where acute and emergency cases are evaluated. Medical records are kept electronically as well as in paper forms. ${ }^{9}$

The overall number of hospital personnel is 1499 (including medical, administrative personnel, and so on). Among them, there are 13 attending general surgeons, 20 attending anesthesiologists, 75 attendings of other surgical specialties and a great number of residents. ${ }^{9}$

\section{Processes}

Administration (location, coverage, governance, funding, development)

Our university hospital covers the city of Thessaloniki and the surrounding area. Trauma patients registered in our database (coded by our general surgery department) represent approximately one-third of trauma patients managed in the hospital. The remaining two-thirds of trauma patients managed in the hospital are not captured by our registry, since during their admission one of the other two general surgery departments covers the trauma/ emergency service. To date, our registry has not received any funding. The present study is the first publication of our registry.

\section{Organization (inclusion criteria, data capture, variables, scores,} analyses)

The inclusion criteria for our registry were defined as trauma patients with at least one traumatic International Classification of Diseases Tenth Revision, to include both minor and severe traumas. Only trauma patients who were evaluated by our surgical team on call (general surgery team, on duty once every 12 days) in the emergency department were recorded in our registry. Patients with pure orthopedic or isolated neurologic injuries seen only by orthopedic or neurosurgical doctors and therefore not evaluated by the general surgery (trauma) team were not included.

A specifically designed computer database was used for data collection. Demographics, mechanisms of injury, injury patterns, field data (ie, vehicle telemetry data, vehicle deformation data, height of falls, and so on), hospital transfer, anatomic descriptions of injuries, vital signs, Glasgow Coma Scale, need for resuscitation (systolic blood pressure (SBP) $<90 \mathrm{~mm} \mathrm{Hg}$ on hospital arrival followed by in-hospital fluid resuscitation or blood transfusion ${ }^{10}$ ) and comorbidities were prospectively recorded after the evaluation of trauma patients in the emergency department. All in-hospital investigations and interventions, length of hospital stay (LOS), outcomes, complications and ICU admissions were retrospectively recorded using hospital records. Cost was also recorded retrospectively. For all trauma hospital admissions, overall non-salary cost for diagnosis and treatment of each trauma patient was provided to our registry by the financial services of the hospital. For all emergency department visits that did not result in hospital admission, all laboratory and radiologic examinations conducted in the emergency room were recorded. The non-salary cost for the diagnosis of each of these patients was calculated as a sum of the costs of the conducted examinations. We were unable to measure the cost of treatment of emergency department visits.

Furthermore, we assessed trauma severity using the Injury Severity Score (ISS). ${ }^{11}$ We evaluated the accuracy of prehospital triage provided by the GNMS. We measured the overtriage (the proportion of trauma patients of ISS $\leq 15$ managed at our level I/II equivalent trauma center) and the undertriage of trauma patients (the proportion of patients of ISS $>15$ who were initially managed at a lower level of healthcare facility and then transferred to our center). ${ }^{12}$ Apart from the typical ISS criteria for overtriage and undertriage mentioned above, we used clinical criteria as well, to determine the patients who actually needed and significantly benefited from receiving specialized trauma management in our facility. Trauma patients who were admitted to the hospital and were hospitalized for more than 48 hours, or received operative management, or whose injury resulted into death were considered accurately triaged. Patients with length of stay $\leq 48$ hours who did not receive operative management and were discharged alive were considered not accurately triaged, as they could have been successfully treated at lower level of healthcare facilities. ${ }^{13-17}$ To determine whether the observed overtriage and undertriage could be reduced by setting certain prehospital criteria for the transfer of trauma patients, the US trauma transfer's (EMS) field triage algorithm was applied to all of our trauma patients. ${ }^{18}$

For the purpose of analyzing the data, the 20 months' study period was divided into two 10-month intervals (2014 interval: from March 1, 2014 to December 31, 2014; 2015 interval: from January 1, 2015 to October 31, 2015). For the description of categorical variables, frequencies and percentages are used. Quantitative variables' normality was tested using Kolmogorov-Smirnov test. Normally distributed variables $\left(\mathrm{p}_{\mathrm{K}-\mathrm{S}}>0.05\right)$ are described as mean $\pm \mathrm{SD}$ and not normally distributed variables $\left(\mathrm{p}_{\mathrm{K}-\mathrm{S}}<0.05\right)$ as median, range: minimum-maximum. The categorical variables were compared by the $\chi^{2}$ test and the normally distributed continuous variables by the independent t-test. Continuous variables with irregular distribution were analyzed by the Mann-Whitney test. $\mathrm{P}<0.05$ was considered statistically significant. Statistical analysis software package SPSS Statistics V.20.0 (IBM) was used for the analysis.

\section{RESULTS}

\section{Demographic information, mechanisms of injury and their} characteristics

The study population was $\mathrm{n}_{\text {total }}=2320$ trauma patients $\left(\mathrm{n}_{2014}=1128\right.$ and $\left.\mathrm{n}_{2015}=1192\right)$. The trauma patients included in our cohort were young $(48 \pm 21$ years old). Falls accounted for the majority of traumas (843 patients-36.3\%) followed by RTCs (539 patients-23.2\%). Traumas caused by RTCs had 
Table $1 \mathrm{~A}$ Overall overtriage and undertriage rates in our trauma patients

\begin{tabular}{|c|c|c|c|c|}
\hline & $\begin{array}{l}\text { Patients transferred } \\
\text { directly to our hospital } \\
\text { (by any means of } \\
\text { transport) }\end{array}$ & $\begin{array}{l}\text { Patients primarily evaluated } \\
\text { in a lower level of } \\
\text { healthcare facility and then } \\
\text { transferred to our hospital } \\
\text { (by any means of transport) }\end{array}$ & Overall overtriage (0) & $\begin{array}{l}\text { Overall } \\
\text { undertriage }(\mathrm{U})\end{array}$ \\
\hline ISS $\leq 15$ & $n_{1}=2236$ & $\mathrm{n}_{2}=8$ & $0=\left(n_{1}+n_{2}\right) / n_{\text {total }}=2244 / 2320=96.7 \%$ & $\mathrm{U}=\mathrm{n}_{4} / \mathrm{n}_{\mathrm{total}}=20 / 2320=1 \%$ \\
\hline ISS $>15$ & $\mathrm{n}_{3}=56$ & $n_{4}=20$ & & \\
\hline $\begin{array}{l}L O S \leq 48 \text { hours and non-operative management and } \\
\text { final outcome: hospital discharge }\end{array}$ & $n_{1}=2098$ & $\mathrm{n}_{2}=6$ & $0=\left(n_{1}+n_{2}\right) / n_{\text {total }}=2104 / 2320=90.7 \%$ & $\mathrm{U}=\mathrm{n}_{4} / \mathrm{n}_{\text {total }}=22 / 2320=1 \%$ \\
\hline $\begin{array}{l}\text { LOS }>48 \text { hours or operative management or final } \\
\text { outcome: death }\end{array}$ & $\mathrm{n}_{3}=194$ & $\mathrm{n}_{4}=22$ & & \\
\hline
\end{tabular}

Table 1B Accuracy of the prehospital triage provided by the GNMS

\begin{tabular}{|c|c|c|c|c|c|c|}
\hline & \multicolumn{2}{|c|}{$\begin{array}{l}\text { Patients transferred directly } \\
\text { to our hospital (by the } \\
\text { GNMS) }\end{array}$} & \multicolumn{2}{|c|}{$\begin{array}{l}\text { Patients transferred from a lower } \\
\text { level of healthcare facility to our } \\
\text { hospital (by the GNMS) }\end{array}$} & \multicolumn{2}{|c|}{ GNMS overtriage (0) } \\
\hline ISS $\leq 15$ & \multicolumn{2}{|l|}{$n_{1}=121$} & \multicolumn{2}{|l|}{$\mathrm{n}_{2}=6$} & \multicolumn{2}{|c|}{$0=n_{1} /\left(n_{1}+n_{3}\right)=121 / 159=76.1 \%$} \\
\hline $\begin{array}{l}\text { LOS } \leq 48 \text { hours and non-operative management and final outcome: hospital } \\
\text { discharge }\end{array}$ & al $n_{1}=90$ & & $n_{2}=4$ & & \multirow{2}{*}{\multicolumn{2}{|c|}{$0=n_{1} /\left(n_{1}+n_{3}\right)=90 / 159=56.6 \%$}} \\
\hline$L O S \geq 48$ hours or operative management or final outcome: death & \multicolumn{2}{|l|}{$\mathrm{n}_{3}=69$} & \multicolumn{2}{|l|}{$n_{4}=22$} & & \\
\hline & $\begin{array}{l}\text { Met the EMS } \\
\text { algorithm criteria }\end{array}$ & \multicolumn{2}{|c|}{$\begin{array}{l}\text { Did not meet the EMS } \\
\text { algorithm criteria }\end{array}$} & Overtriage (0) & & Undertriage $(U)$ \\
\hline ISS $\leq 15$ & $n_{1}=1036$ & \multicolumn{2}{|l|}{$\mathrm{n}_{2}=1208$} & \multirow{2}{*}{\multicolumn{2}{|c|}{$0=n_{1} / n_{\text {total }}=1036 / 2320=44.6 \%$}} & $U=n_{4} / n_{\text {total }}=1 / 2320=0 \%$ \\
\hline ISS $>15$ & $\mathrm{n}_{3}=75$ & \multicolumn{2}{|l|}{$n_{4}=1$} & & & \\
\hline $\begin{array}{l}\text { LOS } \leq 48 \text { hours and non-operative management and final outcome: } \\
\text { hospital discharge }\end{array}$ & $n_{1}=923$ & \multicolumn{2}{|l|}{$\mathrm{n}_{2}=1181$} & $0=n_{1} / n_{\text {total }}=39.7 \%$ & & $\mathrm{U}=\mathrm{n}_{4} / \mathrm{n}_{\text {total }}=28 / 2320=1.2 \%$ \\
\hline
\end{tabular}

EMS, emergency medical service; GNMS, Greek National Medical Services; ISS, Injury Severity Score; LOS, length of hospital stay.

the highest ISS compared with injuries caused by any other mechanism $(\mathrm{p}<0.005)$. For the total of 2320 trauma patients, the mechanisms of injury, the demographic profiles, the injury patterns and the associated severity scores are shown in the online supplementary files 1 and 2 .

\section{Prehospital trauma management and triage}

Out of a total of 2320 trauma patients, only $n=185(8 \%)$ received first aid and hospital transfer by the GNMS. Trauma patients were transferred by the GNMS to our center either directly from the scene $(n=159(6.8 \%))$ or from a lower level of healthcare unit (interfacility transfers) $(n=26(1.1 \%))$. The proportion of trauma patients who were transferred to the hospital by the GNMS decreased between the two study intervals $\left(\mathrm{n}_{2014}=109\right.$ $(9.7 \%)$ vs. $\left.\mathrm{n}_{2015}=76(6.38 \%)\right)(\mathrm{p}<0.05)$. The rest of the patients, constituting the vast majority, came by their own means.

The overall overtriage and undertriage rates for the total of 2230 trauma patients are shown in table 1A. These rates varied depending on the criteria used (clinical vs. ISS criteria). To assess the accuracy of field triage by the GNMS, we also analyzed the overtriage rates of trauma patients who were initially evaluated by the GNMS in the field and then transferred to our center (table 1B). This study was not powered to measure the undertriage rates in the GNMS transferred population. Moreover, we applied the EMS triage algorithm in all of our trauma patients. We showed that the observed overall overtriage could be reduced by more than $50 \%$, if these triage criteria were primarily applied to the trauma patients in the prehospital setting (table 1C). ${ }^{18}$

\section{In-hospital trauma care}

In terms of initial management, all trauma patients were managed according to the advanced trauma life support (ATLS) protocol. All interfacility transfers had their initial assessment in the primary receiving unit. Sixty-two (2.7\%) of our trauma patients were admitted with an SBP $<90 \mathrm{~mm} \mathrm{Hg}$. These patients initially received fluid resuscitation ( $2 \mathrm{~L}$ of crystalloids), followed by blood and product transfusions when deemed necessary. There was no massive transfusion operating protocol in the emergency department at the time. Sixteen (25.8\%) of these patients had eventually an unfavorable outcome.

The vast majority of trauma patients were managed nonoperatively. Out of a total of 2320 trauma patients, 91 (3.9\%) received operative management. Data on our patients' operative management are presented in the online supplementary file 3 . Among our 76 trauma patients with ISS $>15$, thirty-six (47.3\%) received operative management (each patient underwent from one up to five procedures) (damage control), 13 patients (17.1\%) died before reaching the operating theater and 27 (35.5\%) patients received selective non-operative management (SNOM). Among the 36 surgically managed patients, 21 (58.3\%) developed at least one postoperative complication (Clavien-Dindo IIV). Fourteen patients (38.9 \%) were admitted to the Intensive Care Unit (ITU) post-op, either as part of the damage control process or as a result of postoperative complication.

Five hundred $(21.5 \%)$ out of 2320 trauma patients were admitted to a hospital ward. The median LOS was 1 day (range: 1-103 days). In ICU, median LOS was 8 days (range: 1-43). The hospital admissions for the trauma patients included in this cohort are presented in the online supplementary file 4 . 
Table 2A Trauma outcomes

\begin{tabular}{lc}
\hline ( $\mathrm{n}=$ population of the particular group of trauma patients) & Fatalities (mortality \%) \\
\hline Overall trauma mortality (2320) & $29(1.3)$ \\
\hline $\begin{array}{l}\text { Prehospital GNMS mortality (185) } \\
\text { (dead upon arrival patients/GNMS transferred patients) }\end{array}$ & $8(4.3)$ \\
\hline 30 days' surgical mortality (91) & $7(7.7)$ \\
\hline ICU mortality (21) & $8(38.1)$ \\
\hline ISS & $2(0.1)$ \\
\hline 3 (1785) & $0(0)$ \\
\hline $4-8(328)$ & $3(2.3)$ \\
\hline $9-15(131)$ & $1(2.9)$ \\
\hline $16-24(35)$ & $23(56.1)$ \\
\hline $25(41)$ & \\
\hline
\end{tabular}

\section{Outcomes}

Outcomes for the total of 2320 trauma patients are shown in table 2A. Our mortality review is presented in the online supplementary file 5. RTCs were the most common mechanism of injury for 12 of $29(41.4 \%)$ of the trauma fatalities. These were followed by falls-11 of 29 (37.9\%). The RTC fatalities were considerably younger (median age 44 years old (range: 20-89)) compared with the fall fatalities (median age 79 (range: $39-88))(\mathrm{p}<0.005)$. The most prevalent cause of mortality $(11$ of 29 fatalities-37.9\%) in our cohort was the combination of a traumatic brain injury (TBI) with concurrent injuries that resulted in massive hemorrhage or respiratory compromise. Injuries resulting in massive hemorrhage accounted for 6 of 29 fatalities-20.7\%. In 6 of 29 fatalities-20.7\% - their death was attributed in isolated TBI. Eight patients (27.5\%) were dead upon arrival (DUA) and five patients (17.2\%) died in the emergency department before reaching the operating room.

To evaluate the efficacy of the trauma care at our university hospital, we compared our results with the US National Trauma Data Bank, ${ }^{19}$ as well as with the European Trauma Audit and Research Network ${ }^{20}$ (table 2B,C)Given the fact that there is a lack of adjustment for all other factors and variations that influenced outcomes, that the number of our trauma patients is small, and that when comparing mortality rates of traumas of maximal Abbreviated Injury Scale (AIS) score $\geq 4$ statistically significant results could not be reached; there is a bias in these comparisons. No definite conclusions can be drawn; nevertheless, a limited assessment of the current situation can be provided.

\section{Cost analysis of trauma management}

For the total of 2320 trauma patients, overall non-salary cost for trauma management was estimated at approximately €623 140 . The costs accounted for the management of trauma patients across different mechanisms of injury groups are presented in the online supplementary file 6. Approximately $€ 329000(53 \%$ of overall costs) was attributed to the management of traumas caused by RTCs.

Our results presented in table 3 show that the overtriageassociated costs for our hospital range from approximately $€ 121000$ to $€ 315000$ for the study period. $19.4 \%$ to $50.5 \%$ of overall costs for trauma care were dedicated to the management of patients who should not have been triaged to our facility in the first place. Application of the EMS triage algorithm criteria in the prehospital setting could result in reduction of $10.5 \%$ of expenses for our hospital.

\section{DISCUSSION}

\section{Our findings}

In the present study we observed that the youngest of our trauma patients were involved in RTCs. This is in agreement with previous epidemiologic studies. ${ }^{3}$ Approximately one out of two trauma deaths in our cohort were caused by RTCs. RTC fatalities had a median age of 44 years old. The financial burden of RTCs was the greatest among all different mechanisms of injury. Approximately 53\% of overall costs dedicated to trauma management were attributed to managing RTC victims.

Our study indicates that the overtriage of trauma patients to our facility is high (range: $90.7 \%$ to $96.7 \%$ ). Despite our hospital being a tertiary unit, the vast majority of our trauma patients sustained injuries of low severity. Only 9.3\% of them actually needed and benefited significantly from receiving specialized trauma management at our center. This is concerning given the already diminishing resources. The proportion of trauma patients who were evaluated in the prehospital setting and transferred to our hospital by the GNMS was low (8\%) and decreased throughout the study period. The vast majority of patients $(92 \%)$ came to the hospital by their own means. Moreover, the accuracy of the prehospital triage provided by the GNMS was poor. An example of the latter is the fact that two

Table 2B Outcomes of trauma management in our hospital in comparison with the US NTDB

\begin{tabular}{|c|c|c|c|}
\hline & Our trauma registry & US NTDB & \\
\hline Outcomes & $\begin{array}{l}\text { Trauma patients managed in our } \\
\text { hospital } \\
\mathrm{n}(\%)\end{array}$ & $\begin{array}{l}\text { Trauma patients managed in US } \\
\text { trauma centers and non-trauma } \\
\text { centers } \\
\mathrm{n}(\%)\end{array}$ & $P$ value \\
\hline Patients' hospital mortality, with maximal AIS score $\leq 3$ & $6 / 2250(0.3)$ & $134 / 2744(4.9)$ & $<0.001$ \\
\hline Patients' hospital mortality, with maximal AIS score 4 & $3 / 38(7.9)$ & $174 / 1368(12.7)$ & 0.3765 \\
\hline Patients' hospital mortality ${ }^{43}$, with maximal AIS score 5-6 & $20 / 26(76.9)$ & $303 / 931(32.5)$ & 0.1239 \\
\hline
\end{tabular}


Table 2C Outcomes of trauma management in our hospital in comparison with theUS NTDB

\begin{tabular}{lll}
\hline Outcome & Our trauma registry & EuroTARN group \\
\hline & $\mathrm{n}(\%)$ & Range \\
Surviving after trauma of an $I S S>15^{26}$ & $52 / 76(68.4)$ & $24 \%-100 \%$ \\
\hline
\end{tabular}

AIS, Abbreviated Injury Scale; EuroTARN, Trauma Audit and Research Network; GNMS, Greek National Medical Services; ICU, intensive care unit; ISS, Injury Severity Score; NTDB, National Trauma Data Bank.

out of three trauma patients who were transferred by the GNMS from the field to our level I/II equivalent trauma center should not have been triaged there to begin with. The reasons behind the decreasing transfer and overtriage of trauma patients to the highest level of healthcare in the country could be a combination of factors. The lack of specific prehospital triage criteria may have contributed to the increased overtriage rates. Patients and their families may be hesitant to call or wait for the GNMS due to decreased confidence and the limited availability of ambulances and personnel. Most patients arrive at the hospital by private cars, as the hospital is not situated within walking distance from the urban area. In terms of hospital selection, in any given day, trauma patients can choose between primary care facilities, one district general hospital and one tertiary university

Table 3 Cost for diagnosis and management of trauma patients across different injury severity groups and cost of overtriage

\begin{tabular}{|c|c|c|}
\hline $\begin{array}{l}\text { ( } n=\text { population of the particular } \\
\text { group of trauma patients) }\end{array}$ & $\begin{array}{l}\text { Cost for diagnosis and } \\
\text { treatment of patients } \\
\text { (sum of costs) } \\
\text { (\% of overall costs) }\end{array}$ & $\begin{array}{l}\text { Cost per } \\
\text { patient } \\
\text { (mean cost) }\end{array}$ \\
\hline $\begin{array}{l}\text { LOS } \leq 48 \text { hours and non-operative } \\
\text { management and final outcome: } \\
\text { hospital discharge } \\
(n=2104)\end{array}$ & $\begin{array}{l}€ 120761.92 \\
(19.4)\end{array}$ & $€ 57.4$ \\
\hline $\begin{array}{l}\text { LOS }>48 \text { hours or operative } \\
\text { management or final outcome: death } \\
(\mathrm{n}=216)\end{array}$ & $\begin{array}{l}€ 502377.88 \\
(80.6)\end{array}$ & $€ 2325.82$ \\
\hline $\begin{array}{l}\text { Patients (ISS } \leq 15) \text { who should have } \\
\text { been transferred to a level III or IV } \\
\text { trauma center equal } \\
(n=2244)\end{array}$ & $\begin{array}{l}€ 314809.49 \\
(50.5)\end{array}$ & $€ 140.28$ \\
\hline $\begin{array}{l}\text { Patients (ISS }>15) \text { who should have } \\
\text { been transferred to our center (level I/ } \\
\text { II trauma center equal) } \\
(\mathrm{n}=76)\end{array}$ & $\begin{array}{l}€ 308330.31 \\
(49.5)\end{array}$ & $€ 4056.98$ \\
\hline $\begin{array}{l}\text { Patients who fulfilled the EMS triage } \\
\text { algorithm criteria for trauma center } \\
\text { transfer } \\
(\mathrm{n}=1111)\end{array}$ & $\begin{array}{l}€ 557460.63 \\
(89.5)\end{array}$ & $€ 501.76$ \\
\hline $\begin{array}{l}\text { Patients who did not fulfill the EMS } \\
\text { algorithm criteria for trauma center } \\
\text { transfer } \\
(\mathrm{n}=1209)\end{array}$ & $\begin{array}{l}€ 65679.17 \\
(10.5)\end{array}$ & $€ 54.32$ \\
\hline $\begin{array}{l}I S S \leq 3 \\
(n=1785)\end{array}$ & $\begin{array}{l}€ 102619.17 \\
(16.5)\end{array}$ & $€ 57.49$ \\
\hline $\begin{array}{l}4 \leq 1 \mathrm{SS} \leq 8 \\
(\mathrm{n}=328)\end{array}$ & $\begin{array}{l}€ 60476.1 \\
(9.7)\end{array}$ & $€ 184.38$ \\
\hline $\begin{array}{l}9 \leq 1 \mathrm{SS} \leq 15 \\
(\mathrm{n}=131)\end{array}$ & $\begin{array}{l}€ 151714.22 \\
(24.3)\end{array}$ & $€ 1158.12$ \\
\hline $\begin{array}{l}16 \leq 1 S S \leq 24 \\
(n=35)\end{array}$ & $\begin{array}{l}€ 107924.91 \\
(17.3)\end{array}$ & $€ 3083.57$ \\
\hline $\begin{array}{l}I S S \geq 25 \\
(n=41)\end{array}$ & $\begin{array}{l}€ 200405.40 \\
(32.2)\end{array}$ & $€ 4887.94$ \\
\hline
\end{tabular}

EMS, emergency medical service; ISS, Injury Severity Score; LOS, length of hospital stay. hospital when driving in by their own means. It seems that they prefer to be managed at the highest level of healthcare facility in the country that is adequately resourced and staffed, compared with lower level of care facilities which may be lacking resources. Our hospital's reputation has also a role to play, since it is one of the leading academic units in Northern Greece. This practice leads to a vicious circle: transformation of the structure of the national injury pyramid and disproportionate rationing of resources among the different levels of healthcare. ${ }^{21}$ Our study demonstrated that the application of the EMS criteria as a field triage decision scheme for all trauma patients would result in a reduction of more than $50 \%$ of the observed overtriage rate. A significant proportion of the personnel's workload would be also reduced as well.

Regarding the financial impact of the findings mentioned above, the cost-benefit ratio of the existing national trauma management strategy was very high. Application of the EMS algorithm criteria in the field on all the trauma patients could result in $10.5 \%$ reduction of costs for tertiary centers. More effective prehospital triage (identifying more severely injured patients (ISS > 15) and treating them at the appropriate level of healthcare) can lead to a decrease of $50.5 \%$ in the expenditure dedicated to trauma management in the third level of healthcare in the country. These resources could be better allocated to the organization of an inclusive trauma system. Considering the ratio of personnel to patients, and consequently the total salary costs are lower in primary and secondary healthcare structures, in comparison with the tertiary units, a significant reduction in the national expenditure dedicated to trauma could be achieved by decreasing the observed overtriage of patients at the highest level of healthcare in the country. 223

In terms of in-hospital major trauma care, we observed that one out of four major trauma patients who were admitted with an $\mathrm{SBP}<90 \mathrm{~mm} \mathrm{Hg}$ had an unfavorable outcome. This, in combination with the high incidence of prehospital deaths (DUAs) and emergency department deaths, raises concerns about the system's capacity to enable timely interventions in patients with massive hemorrhage. This finding can be also attributed to the delayed onset of the resuscitation process and the absence of effective resuscitation operating protocols (ie, massive transfusion protocol). More importantly, it demonstrates the absence of streamlined care processes from the prehospital setting to the emergency department and then to the operative theater and the ICU. There is no pre-established coordination between these services.

\section{Comparison to national and international registries}

In a previous systematic review of our team, we found that over the last decades a number of registries have been established across Greece. ${ }^{4} 52124-42$ In terms of demographics and mechanisms of injury few things have changed for Greek trauma over the past decades. As recorded in our study, Greek trauma patients are mostly young, and the leading mechanisms of injury are RTCs and falls. ${ }^{4} 52125-41$ Our major finding regarding the need for improved prehospital trauma patient management and 
transfer is not a new problem for the Greek healthcare system. ${ }^{24}$ Review of these registry publications revealed that throughout the last decades the percentage of injuries of minor or moderate severity (ISS $<15$ ) that are managed at tertiary-level healthcare facilities has increased. ${ }^{21} 353641$ This increase is in agreement with our findings about the inefficacy of the prehospital trauma triage, especially during the years of financial crisis. In terms of in-hospital trauma management, Drimousis et al found that ATLS-certified physicians achieve worse outcomes than their non-certified colleagues when managing trauma patients. ${ }^{38}$ This finding, in combination with the ineffectiveness in the management of critical traumas and massive hemorrhage observed in our study, raises concerns regarding how the current system's structure capacitates the proper ATLS implementation in clinical practice. Future studies should determine the proportion of ATLS-certified physicians among all physicians involved in trauma management in the country. Local policies and quality improvement efforts are necessary to address the existing obstacles in the proper implementation of the ATLS protocol.

The comparison of our outcomes with international trauma registries suggested that the mortality in our center for ISS $>24$ appears to be higher compared with US/EU trauma centers. To investigate this finding, we performed a mortality review. The combination of TBI with concurrent injuries that resulted in massive hemorrhage was the leading cause of mortality in our unit. Most of these patients died before reaching the operating room or within the first 48 hours. This reflects the lack of leadership and coordination in the trauma service, from the field to the operating room and the ICU. There is fragmentation of care and lack of streamlined processes. This hinders rapid decisionmaking and determination of consistent management plans. On the contrary, the mortality for ISS $<24$ is within the international limits. In patients who respond to resuscitation and have clear management priorities, there is coordination of the care by the relevant specialty. Because the expertise, the resources and the infrastructures are in place, these patients seem to do better. Nevertheless, the number of trauma patients with $15<$ ISS $<24$ included in this cohort is relatively small. Definitive conclusions on the comparative mortality assessment with international registries cannot be reached. In terms of operative management of injuries, one out of two major trauma patients (ISS $>15$ ) received operative management with very good outcomes. This percentage is not low, if one considers that approximately $20 \%$ of our trauma patients deceased either on the field or in the emergency department, before reaching the operative room. One-third of our trauma patients received SNOM rate similar to European and US trauma centers. ${ }^{43-46}$ The non-therapeutic intervention ratio (ie, negative laparotomies) was not recorded in our registry. Future comparative studies are necessary to better investigate the efficacy of the management of major trauma in Greece, and to enable improvement efforts.

The study findings have important clinical implications. Improved road safety programs and precaution measures should be adopted nationally to decrease the occurrence of RTCs and reduce the relevant healthcare expenses. There is also a need for improved organization and efficacy in the primary healthcare and in the GNMS for trauma patient evaluation. In addition, they should play a more active role in the triage of these patients, to increase the cost-effectiveness of trauma management. Definition of specific criteria for the field triage of the injured is pivotal for decreasing the observed overtriage at the highest level of healthcare in the country. To have a more organized, protocol-based approach, consideration should be given, similar to other countries, to having trauma surgery as an independent specialty, which would mean advanced training and accreditation processes. ${ }^{47}$ This might likely result in leadership initiatives on the field and quality improvement efforts to streamline care processes, develop local trauma protocols and overcome barriers in their implementation. The trauma level for each hospital should be accurately defined, reflecting the capabilities of each center in accepting and treating trauma patients. The current operating system can serve as platform for regionalization of the trauma service. The development of an inclusive trauma network has the potential to result in advanced service coordination and better outcomes. There are transferable lessons in the London experience. ${ }^{48}$ Most importantly, this study shows the need to develop a national consensus and to establish a Greek National Trauma Database to credibly measure the impact of trauma in our country and enable our system to make chances for improvement.

\section{Limitations}

The present study has several limitations. Data were collected through our single center registry and refer to a group of trauma patients who are managed during a certain period of time at a university hospital in Greece. During data collection, we were unable to record the cost of treatment of trauma patients who were evaluated in the emergency department but were not admitted to a hospital department, so we only recorded the cost of diagnosis of these trauma patients. Undertriage rates in the GNMS transferred population could not be estimated by our study, because it was not recorded during data collection whether the trauma patients who received an interfacility transfer were initially evaluated by the GNMS (in the field). During data analysis, when comparing our results with international data, it was not possible to adjust outcomes for variations and due to the small number of patients of maximal AIS score $\geq 4$ in our study, statistically significant results could not be reached.

\section{CONCLUSIONS}

In conclusion, RTCs pose a significant financial burden for the Greek healthcare system. The prehospital trauma triage is ineffective and leads to disproportionate rationing of resources among levels of healthcare in the country. Cost-effectiveness of trauma management can be improved through increasing patients' triage precision. Future studies are needed to investigate the efficacy of the management of polytrauma patients in Greece, and to enable improvement efforts.

Contributors APr, GT, and VP designed the study. APr, AT, APa and APi collected the data. APr, AT, APa and APi performed the data analysis. APr drafted the article. $A P r, G T$ and VP critically revised the article.

Funding The authors have not declared a specific grant for this research from any funding agency in the public, commercial or not-for-profit sectors.

Competing interests None declared.

Patient consent for publication Not required.

Ethics approval This study was conducted in accordance with the ethical standards of the Papageorgiou General Hospital Research Committee and with the 1964 Helsinki Declaration and its later amendments.

Provenance and peer review Not commissioned; externally peer reviewed.

Data availability statement Data are available upon reasonable request. Anonymized non-identifiable data are available through the hospital registry (contact: bnpap@med.auth.gr for information/reuse).

Open access This is an open access article distributed in accordance with the Creative Commons Attribution Non Commercial (CC BY-NC 4.0) license, which permits others to distribute, remix, adapt, build upon this work non-commercially, and license their derivative works on different terms, provided the original work is 
properly cited, appropriate credit is given, any changes made indicated, and the use is non-commercial. See: http://creativecommons.org/licenses/by-nc/4.0/.

ORCID iD

Apostolos Prionas http://orcid.org/0000-0002-7867-9266

\section{REFERENCES}

1 World health Organization. The top 10 causes of death. 2017. http://www.who.int/ mediacentre/factsheets/fs310/en/ (Mar 2017).

2 World Health Organization. Progress in prevention of injuries in WHO European region: Greece. 2016. http://www.euro.who.int/_data/assets/pdf_file/0003/98715/ Greece.pdf?ua=1 (Feb 2016).

3 O'Reilly GM, Cameron PA, Joshipura M. Global trauma registry mapping: a scoping review. Injury 2012;43:1148-53.

4 Theodorou D, Toutouzas K, Drimousis P, Larentzakis A, Kleidi E, Georgiou G, Gymnopoulos D, Kandylakis S, Theodoraki ME, Katsaragakis S, et al. Emergency room management of trauma patients in Greece: preliminary report of a national study. Resuscitation 2009;80:350-3.

5 Katsaragakis S, Drimousis PG, Kleidi ES, Toutouzas K, Lapidakis E, Papadakis G, Daskalakis K, Larentzakis A, Theodoraki ME, Theodorou D, et al. Interfacility transfers in a non-trauma system setting: an assessment of the Greek reality. Scand J Trauma Resusc Emerg Med 2010;18:14.

6 OECD and the European Commission. Health at a Glance: Europe. 2014. http://www. oecd.org/health/health-at-a-glance-europe-23056088.htm (Feb 2016).

7 Anderson C, Squires D. U.S Health Care from a Global Perspective: Spending, Use of Services, Prices, and Health in 13 Countries. 2015. http://www.commonwealthfund. org/publications/issue-briefs/2015/oct/us-health-care-from-a-global-perspective. Accessed (Mar 2016)

8 Hellenic Statistical Authority. Health Expenditures- System of Health Accounts. www. statistics.gr (Feb 2016)

9 http://www.papageorgiou-hospital.gr/c1/index.php?lang=el.

10 American College of Surgeons. Atls student course manual: advanced trauma life support. 9th edn, 2012

11 Moore EE, Moore FA. American association for the surgery of trauma organ injury scaling: 50th anniversary review article of the Journal of trauma. J Trauma 2010:69:1600-1.

12 Lossius HM, Rehn M, Tjosevik KE, Eken T. Calculating trauma triage precision: effects of different definitions of major trauma. J Trauma Manag Outcomes 2012;6:9.

13 Lynch KT, Essig RM, Long DM, Wilson A, Con J. Nationwide secondary overtriage in level 3 and level 4 trauma centers: are these transfers necessary? I Surg Res 2016:204:460-6.

14 Osen HB, Bass RR, Abdullah F, Chang DC. Rapid discharge after transfer: risk factors, incidence, and implications for trauma systems. J Trauma 2010;69:602-6.

15 Ciesla DJ, Sava JA, Street JH, Jordan MH. Secondary overtriage: a consequence of an immature trauma system. J Am Coll Surg 2008:206:131-7.

16 Sorensen MJ, von Recklinghausen FM, Fulton G, Burchard KW. Secondary overtriage: the burden of unnecessary interfacility transfers in a rural trauma system. JAMA Surg 2013;148:763-8.

17 Con J, Long D, Sasala E, Khan U, Knight J, Schaefer G, Wilson A. Secondary overtriage in a statewide rural trauma system. J Surg Res 2015;198:462-7.

18 Sasser SM, Hunt RC, Faul M, Sugerman D, Pearson WS, Dulski T, Wald MM, Jurkovich GJ, Newgard CD, Lerner EB, et al. Guidelines for field triage of injured patients: recommendations of the National expert panel on field triage, 2011. MMWR Recomm Rep 2012;61:1-20.

19 MacKenzie EJ, Rivara FP, Jurkovich GJ, Nathens AB, Frey KP, Egleston BL, Salkever DS, Scharfstein DO. A national evaluation of the effect of trauma-center care on mortality. N Engl J Med 2006;354:366-78.

20 Edwards A, Di Bartolomeo S, Chieregato A, Coats T, Della Corte F, Giannoudis P, Gomes E, Groenborg H, Lefering R, Leppaniemi A, et al. A comparison of European trauma registries. The first report from the EuroTARN group. Resuscitation 2007;75:286-97.

21 Kanakaris N. Quality control of trauma in GREECE- Hellenic trauma database: PhD National Kapodistrian University of Athens Medical School, 2014.

22 Newgard CD, Staudenmayer K, Hsia RY, Mann NC, Bulger EM, Holmes JF, Fleischman R, Gorman K, Haukoos J, McConnell KJ. The cost of overtriage: more than onethird of low-risk injured patients were taken to major trauma centers. Health Aff 2013:32:1591-9.

23 DeKeyser FG, Paratore A, Seneca RP, Trask A. Decreasing the cost of trauma care: a system of secondary inhospital triage. Ann Emerg Med 1994;23:841-4.

24 Papadopoulos IN, Bukis D, Karalas E, Katsaragakis S, Stergiopoulos S, Peros G, Androulakis $\mathrm{G}$. Preventable prehospital trauma deaths in a Hellenic urban health region: an audit of prehospital trauma care. J Trauma 1996;41:864-9.
25 Papadopoulos IN, Kanakaris N, Triantafillidis A, Stefanakos J, Kainourgios A, Leukidis C. Autopsy findings from 111 deaths in the 1999 Athens earthquake as a basis for auditing the emergency response. Br J Surg 2004;91:1633-40.

26 Kanakaris N, Papadopoulos I, Triantafillidis A. Motor vehicle Collisions-Letha trauma-Pelvic girdle involvement. The Greek reality. European Journal of Trauma 2006:32:92-3.

27 Kanakaris N, Papadopoulos I, Triantafillidis A, et al. " Lethal MVA Spinal Injury.". European Journal of Trauma 2006:32:73.

28 Papadopoulos I, Kanakaris N, Triantafillidis A, et al. "Autopsy findings of Pulmonary Thromboembolism in Trauma patients." . European Journal of Trauma 2006;32:148.

29 Papadopoulos IN, Kanakaris N, Bonovas S, Triantafillidis A, Garnavos C, Voros D, Leukidis C. Auditing 655 fatalities with pelvic fractures by autopsy as a basis to evaluate trauma care. J Am Coll Surg 2006:203:30-43.

30 Christodoulou C, Papadopoulos IN, Douzenis A, Kanakaris N, Leukidis C, Gournellis R, Vlachos K, Papadopoulos FC, Lykouras L. Seasonality of violent suicides in the Athens greater area. Suicide Life Threat Behav 2009;39:321-31.

31 Papadopoulos IN, Bonovas S, Kanakaris NK, Konstantiadou I, Nikolopoulos G, Konstantoudakis G, Leukidis C. Motor vehicle collision fatalities involving alcohol and illicit drugs in Greece: the need for management protocols and a reassessment of surveillance. Addiction 2010;105:1952-61.

32 Papadopoulos IN, Kanakaris NK, Bonovas S, Konstantoudakis G, Petropoulou K Christodoulou S, Kotsilianou O, Leukidis C, et al. Patients with pelvic fractures due to falls: a paradigm that contributed to autopsy-based audit of trauma in Greece. J Trauma Manag Outcomes 2011:5:1-15.

33 Papadopoulos IN, Bonovas S, Kanakaris NK, Nikolopoulos G, Kotsilianou O, Konstantoudakis G, Leukidis C. Alcohol and psychoactive drugs increased the pre-hospital mortality in 655 fall-related fatalities in Greece: a call for management protocols. Injury 2012:43:1522-6.

34 Papadopoulos IN, Kanakaris NK, Danias N, Sabanis D, Konstantudakis G, Christodoulou S, Bassiakos YC, Leukidis C. A structured autopsy-based audit of 370 firearm fatalities: contribution to inform policy decisions and the probability of the injured arriving alive at a hospital and receiving definitive care. Accid Anal Prev 2013:50:667-77.

35 Markogiannakis H, Sanidas E, Messaris E, Koutentakis D, Alpantaki K, Kafetzakis A, Tsiftsis D. Predictors of in-hospital mortality of trauma patients injured in vehicle accidents. Ulus Travma Acil Cerrahi Derg 2008;14:125-31.

36 Markogiannakis H, Sanidas E, Messaris E, Koutentakis D, Alpantaki K, Kafetzakis A, Tsiftsis D. Motor vehicle trauma: analysis of injury profiles by road-user category. Emerg Med J 2006;23:27-31.

37 Kastanaki AE, Kranioti EF, Papavdi A, Theodorakis PN, Michalodimitrakis M. Suicide by firearms on the island of Crete: a 9-year record. Crisis 2010;31:43-52.

38 Drimousis PG, Theodorou D, Toutouzas K, Stergiopoulos S, Delicha EM, Giannopoulos $P$, Larentzakis A, Katsaragakis $S$. Advanced trauma life support certified physicians in a non trauma system setting: is it enough? Resuscitation 2011:82:180-4.

39 Tsatsanidis GV, Minopoulos GI, Liratzopoulos ND, Papathanasiou JV, Simopoulos KE. The structure of trauma patients, its management and health care in the university hospital of Alexandroupolis. Folia Med 2014;56:50-5.

40 Lanitis S, Kontovounisios C, Zafeiriadou P, Sgourakis G, Karkoulias K, Armoutides V, Papaconstandinou T, Karaliotas C. Epidemiology and management of trauma patients in a Greek Multispecialty hospital in the absence of a dedicated trauma center. Eur J Trauma Emerg Surg 2013;39:369-74.

41 Gardikou V, Christopoulos C, Stamatiou K. "The Multi-injured Patient in the Urban Environment". Achaiki latriki 2015;34:1.

42 Prionas A, Toulias A, Tsoulfas G, Papadopoulos VN, et al. Measuring the impact of trauma in Greece: a systematic review of Greek trauma registries. is quality improvement achievable? Hellenic J Surg 2018;90:75-84.

43 Maegele M, Lefering R, Sakowitz O, Kopp MA, Schwab JM, Steudel WI, Unterberg A Hoffmann R, Uhl E. Marzi I: the incidence and management of moderate to severe head injury — retrospective analysis of data from the trauma register of the German trauma Society. Dtsch Arztebl Int 2019;116:167-73.

44 Goedecke M, Kühn F, Stratos I, Vasan R, Pertschy A, Klar E. No need for surgery? patterns and outcomes of blunt abdominal trauma. Innov Surg Sci 2019;4:100-7.

45 Pande R, Saratzis A, Winter Beatty J, Doran C, Kirby R, Harmston C. Contemporary characteristics of blunt abdominal trauma in a regional series from the UK. Ann $R$ Coll Surg Eng/ 2017;99:82-7.

46 Stawicki SPA. Trends in nonoperative management of traumatic injuries - A synopsis. Int J Crit IIIn Inj Sci 2017;7:38-57.

47 Strumwasser A, Grabo D, Inaba K, Matsushima K, Clark D, Benjamin E, Lam L, Demetriades $D$. Is your graduating general surgery resident qualified to take trauma call? A 15-year appraisal of the changes in general surgery education for trauma. J Trauma Acute Care Surg 2017;82:470-80.

48 Cole E, Lecky F, West A, Smith N, Brohi K, Davenport R, . ELoTS Study Collaborators. The impact of a Pan-regional inclusive trauma system on quality of care. Ann Surg 2016;264:188-94 